American Journal of Biochemistry and Biotechnology 3 (2): 92-102, 2007

ISSN 1553-3468

(C) 2007 Science Publication

\title{
The NifX Protein is Involved in the Final Stages of FeMo-cofactor Transport to the MoFe Protein
}

\author{
Surobhi Lahiri, Byron Cole, Lakshmi Pulakat and Nara Gavini \\ Department of Biological Sciences, Mississippi State University, Mississippi State, MS 39762, USA
}

\begin{abstract}
The nitrogenase enzyme catalyzes the reduction of dinitrogen to ammonia and is composed of the $\mathrm{Fe}$ and MoFe proteins. The iron molybdenum cofactor (FeMo-co) of the MoFe protein is the site of active substrate reduction. The NifX protein has also been suggested to have a role in the FeMo-co synthesis, although its exact role is still open to investigation. We attempted to understand the role of NifX by determining the specific interactions it may have with other Nif proteins involved in FeMo-co synthesis, such as NifD, NifK, NifN, NifDK and NifH. Using the BacterioMatch Two-Hybrid System, a translationally fused construct of NifX with the N-terminal $\alpha$-RNAP of the pTRG target vector was made and its interaction was tested with the NifDK fusion protein, translationally fused to the $\lambda \mathrm{CI}$ of the $\mathrm{pBT}$ vector. The strength of the interaction, as determined by measuring the $\beta$-galactosidase activity, demonstrated that direct protein-protein interaction exists between NifDK and NifX proteins; the extent of interaction between NifK and NifX proteins was much higher than between NifD and NifX, when individually tested; also, reduced interaction was found between NifH and NifX.
\end{abstract}

Key words: FeMo-cofactor, NifX, bacteriomatch two-hybrid system, protein-protein interactions

\section{INTRODUCTION}

The nitrogenase enzyme converts the atmospheric nitrogen into a bioavailable form, thus serving as a focal point in structural, molecular and biochemical studies $^{[1-3]}$. The molybdenum containing nitrogenases (Mo-nitrogenases) are the best characterized metalloenzymes and are composed of two oxygen sensitive metalloproteins known as the MoFe protein (NifDK) and the Fe protein (NifH). The MoFe protein contains two types of metal centers, the P-cluster and the iron-molybdenum cofactor $(\mathrm{FeMoco})^{[4]}$. The FeMoco is the active site of the Mo-nitrogenase and composed of seven $\mathrm{Fe}$, nine $\mathrm{S}$, one $\mathrm{Mo}$, one homocitrate and one atom of unidentified nature ${ }^{[5,6]}$. It is entirely situated in the NifD subunit which provides one Cys and one His ligand for bonding with the $\mathrm{Fe}$ and Mo ends of its metallostructure respectively. Considerable research efforts have been directed towards understanding the biosynthesis and sequential assembly of the FeMoco and it is known that various nitrogen fixation (nif) genes play a role in the biosynthesis, assembly, transport and insertion of the FeMocofactor $^{[7,8]}$. The products of the nifB, nifV, nifQ, nifH, nifE, nifN, nif $X$ and naf $Y$ genes have been identified to be the prime players in these processes ${ }^{[9-11]}$.
Two different pathways for the biosynthesis of FeMoco could be pictured: (i) the FeMoco units could be sequentially assembled on the apo-MoFe or (ii) the FeMoco could be separately synthesized and then inserted onto the apo-MoFe protein. However, as established by past experiments, the second pathway proved to be accurate, whereby mutations in either nifB, nif $N$ or nifE, that are involved in the biosynthesis of FeMoco, resulted in very low molybdenum accumulation and a molybdenum-free $\mathrm{MoFe}$ protein $^{[12,13]}$. Thus it was demonstrated that an active FeMoco could be synthesized in the absence of the MoFe protein and that the FeMoco could accumulate on a different protein, such as an intermediate in the normal FeMoco biosynthetic pathway ${ }^{[13]}$. A significant progress in determining the protein components involved in the biosynthesis of the FeMoco ensued from the development of an in vitro assay for FeMoco biosynthesis ${ }^{[14]}$. This assay utilized a mixture of extracts prepared from mutant strains that presumably had complementary defects in FeMoco biosynthesis, so as to attempt reconstitution of the MoFe protein activity. This application proved immensely useful in recognizing NifH, NifEN and NifV as some of the key players in the FeMoco biosynthetic pathway and helped in the purification of an apo-MoFe protein ${ }^{[13-17]}$. As understood from research conducted thus far, a stepwise

Corresponding Author: Nara Gavini, Department of Biological Sciences, Mississippi State University, Mississippi State, MS 39762 
overview of the events involved in the biosynthesis and assembly of the FeMoco includes: (i) the formation of an $\mathrm{Fe} / \mathrm{S}$ core of the FeMoco (designated NifB-co) by $\mathrm{NifB}^{[18,19]}$ (ii) transfer of the NifB-co to the $\alpha_{2} \beta_{2}$ tetrameric scaffold NifEN protein ${ }^{[20,21]}$ (iii) maturation of the FeMoco and apo-MoFe protein by the combined action of the Fe protein and $\mathrm{MgATP}^{[17,22,23]}$ and further processing of the NifB-co on NifEN by an unknown mechanism, forming the completed FeMoco (iv) the final transfer of the fully formed FeMoco to a nafYencoded protein, called wwhich is a FeMoco carrier that aids in the insertion of the FeMoco into the apo$\mathrm{MoFe}^{[24,25]}$. In addition to these major events, a few other important steps are also involved in the entire process. The NifU and NifS proteins participate in the initial $\mathrm{Fe} / \mathrm{S}$ mobilization ${ }^{[26,27]}$, the NifQ protein is involved in an early stage of the metal core formation and found to be essential in Mo-limiting conditions ${ }^{[28]}$, the NifV protein contributes the homocitrate component of the FeMoco ${ }^{[29]}$ and the NifX protein probably functions as an 'escort' protein that delivers the FeMoco or its precursors from one assembly site to another or plays a role in specifying the organic acid moiety of FeMoco $^{[30,31]}$.

Although a wealth of data is available to support the functions of the NifU, NifS, NifB, NifEN, NifH and $\mathrm{NifV}$, more research is required to explain the role of the NifX protein. The nif $X$ gene is located downstream of the nifEN genes, such that nifENX are transcribed as a single transcription unit. The NifX protein is comprised of 158 amino acids and is a $\sim 17 \mathrm{kDa}$ protein $^{[32]}$. The small molecule binding domain (SMBD) of NifX has widespread phyletic distribution and therefore indicates an ancient origin or extensive dissemination by horizontal transfer during evolution, or both ${ }^{[33]}$. Eventhough a deletion in the nif $X$ gene did not show a significant effect on the nitrogenase activity in vivo, the purified NifX protein was shown to be important in the stimulation of FeMoco synthesis in an in vitro FeMoco synthesis reaction ${ }^{[10,34]}$. The NifX, NifB, NifY and NafY are most likely related proteins, owing to their overall sequence similarity and the presence of a FeMoco binding 'core domain' in all of them ${ }^{[35]}$. This conserved family of proteins has a $\beta_{1}-\beta_{2}-\beta_{3}-\alpha_{1}-\alpha_{2}-\beta_{4}-\alpha_{3}-\beta_{5}-\alpha_{4}-\alpha_{5}$ fold in its core domain, a polarized surface charge distribution and shows considerable similarity with the ribonuclease $\mathrm{H}$ family ${ }^{[35]}$. In NifB, the NIFX SMBD is fused with a biotin-synthase-like, metal-dependent catalytic domain and in the archaeal MTH1172 protein, it is fused to a cation transporter ${ }^{[33]}$. Previous studies in Azotobacter vinelandii have identified certain important molecular interactions of the NifX protein with the other Nif proteins involved in the FeMoco biosynthesis/transport pathway; mainly it was shown that NifX binds to the NifB-co and also a FeMoco precursor from the NifEN complex ${ }^{[10,30]}$.

Clearly, a protein-protein interaction based study of NifX with other FeMoco maturation proteins would enhance our insight into the role of NifX and may help to solve unanswered questions regarding its position in the FeMoco biosynthetic pathway. We therefore studied the interactions of NifX with some of the other relevant proteins participating in FeMoco biosynthesis /assembly/transport /insertion, so as to deduce the entire sequence of events involved. We determined the specific interactions of NifX with NifB, NifH, NifN, NifD, NifK, NifDK (fusion) and NafY using the BacterioMatch Two-Hybrid $\quad$ System $^{\text {TM[36,37]. }}$ Accordingly, a translationally fused construct of NifX with the N-terminal $\alpha$-RNAP of the pTRG target vector was made and its interaction was tested with the above proteins, each translationally fused to the $\lambda \mathrm{CI}$ of the pBT vector. The strength of the interactions, as determined by measuring the $\beta$-galactosidase activity, demonstrated that direct protein-protein interaction exists between (i) NifB and NifX (ii) NafY and NifX and (iii) NifDK (fusion) and NifX (iv) NifK and NifX. The extent of interaction between NifK and NifX proteins was much higher than between NifD and NifX, when individually tested; also, unsubstantial interaction was found between NifH and NifX and NifN and NifX. Our results present a new dimension to the FeMoco biosynthesis pathway with reference to the role played by NifX. We propose that the NifX performs overlapping functions in the FeMoco biosynthetic pathway wherein it may either function as a transporter of the FeMoco precursor to the NafY protein or directly assist in the insertion of the completed FeMoco into the apo-MoFe protein.

\section{MATERIALS AND METHODS}

Strains, plasmids and growth conditions: The bacterial strains and plasmids used in this study are described in Table 1. E. coli strains were normally grown at $37^{\circ} \mathrm{C}$ in $2 \mathrm{YT}$ media ${ }^{[38]}$. Ampicillin, chloramphenicol and tetracycline were used to a final concentration of 50,34 and $5 \mu \mathrm{g} \mathrm{mL}{ }^{-1}$, respectively, wherever the selection was made.

General molecular techniques: Restriction enzymes were purchased from Promega (Madison, WI). DNA sub-cloning, plasmid DNA isolations, restriction 
Am. J. Biochem. \& Biotechnol., 3 (2): 96-106, 2007

Table 1: Bacterial Strains and Plasmids used in this study

\begin{tabular}{|c|c|c|}
\hline Strain/plasmid & Relevant characteristics and description & Source/reference \\
\hline $\begin{array}{l}\text { Escherichia coli } \\
\text { TOP10 }\end{array}$ & 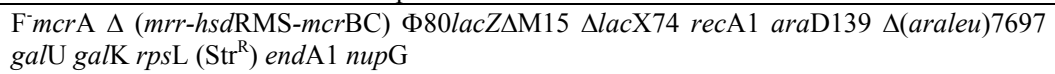 & Invitrogen, $\mathrm{CA}$ \\
\hline $\begin{array}{l}\text { Escherichia coli XL-1 } \\
\text { Blue }\end{array}$ & 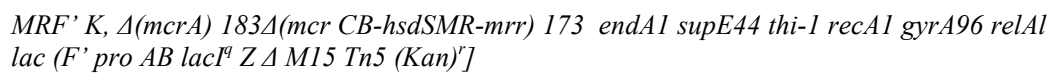 & Stratagene, CA \\
\hline PCR 2.1 TOPO & $A m p^{r}, K^{r}{ }^{r},(3.9 \mathrm{~kb})$, used for direct cloning of PCR products, lacZa fragment, MCS, M13 & Invitrogen, CA \\
\hline pBT & $\mathrm{Cm}^{R}, 52 \mathrm{bp} M C S, 3.2 \mathrm{~kb}$ size, $M C S, p 15 \mathrm{~A}$ origin of replication, lac-UV5, $\lambda \mathrm{cl}$ ORF & Stratagene, CA \\
\hline pTRG & $\begin{array}{l}T e t^{R}, 60 \mathrm{bp} M C S, 4.4 \mathrm{~kb} \text { size, MCS, lac-UV5 promoter, CoE1 origin of replication, } R N A P \alpha \\
\text { ORF }\end{array}$ & Stratagene, CA \\
\hline pBG1866 & $\begin{array}{l}\text { Derivative of } \mathrm{pBT} \text { in which } 879 \mathrm{bp} \text { nifH fragment was cloned into BamHI site of } \mathrm{pBT} \text { to } \\
\text { generate an in-frame } \lambda \mathrm{CI} \text { :NifH translation fusion }\end{array}$ & [39] \\
\hline pBG1713 & $\begin{array}{l}\text { Derivative of } \mathrm{pBT} \text { in which } 3062 \mathrm{bp} \text { orf corresponding to nifD-K fusion gene from XhoI- } \\
E c o R V \text { digested pBG } 1712 \text { was cloned into SmaI-XhoI site of } \mathrm{pBT} \text { bait vector to generate an } \\
\text { in-frame } \lambda \text { CI:NifD-K translation fusion }\end{array}$ & [40] \\
\hline pBG1716 & $\begin{array}{l}\text { Derivative of pTRG in which } 2335 \text { bp nifKTY fragment was cloned into the BamHI site of } \\
\text { pTRG to generate an in-frame } \alpha \text {-RNAP: NifX translation fusion }\end{array}$ & {$[40]$} \\
\hline pBG1718 & $\begin{array}{l}\text { Derivative of pBT in which } 2335 \text { bp nifKTY fragment released from BamHI digested } \\
\text { pBG1715 was cloned into BamHI site of pBT to generate an in-frame } \lambda \text { CI NifKTY } \\
\text { translation fusion }\end{array}$ & [40] \\
\hline pBG1777 & $\begin{array}{l}\text { Derivative of PCR } 2.1 \text { TOPO in which } 1377 \text { bp nif } N \text { fragment was cloned directly after PCR } \\
\text { using appropriate olignonucleotides designed with flanking EcoRI-BamHI sites }\end{array}$ & This study \\
\hline pBG1778 & $\begin{array}{l}\text { Derivative of } \mathrm{pBT} \text { in which } 1377 \mathrm{bp} \text { nifN fragment released from EcoRI-BamHI digested } \\
\text { pBG1777 was cloned into the EcoRI-BamHI site of pBT to generate an in-frame } \lambda C I \text { :NifN } \\
\text { translation fusion }\end{array}$ & This study \\
\hline pMH5014 & $\begin{array}{l}\text { Derivative of PCR } 2.1 \text { TOPO in which } 1-300 \text { bp nif } X \text { fragment was cloned directly after } \\
\text { PCR using appropriate olignonucleotides designed with flanking BamHI-EcoRI sites }\end{array}$ & This study \\
\hline pBG1786 & $\begin{array}{l}\text { Derivative of pTRG in which } 1-300 \text { bp nif } X \text { fragment released from BamHI-EcoRI digested } \\
\text { pMH5014 was cloned into the BamHI-EcoRI site of pTRG to generate an in-frame } \alpha \text { - } \\
\text { RNAP:N1NifX translation fusion }\end{array}$ & This study \\
\hline pBG1782 & $\begin{array}{l}\text { Derivative of PCR } 2.1 \text { TOPO in which } 150-477 \text { bp nif } X \text { fragment was cloned directly after } \\
\text { PCR using appropriate olignonucleotides designed with flanking BamHI-EcoRI sites }\end{array}$ & This study \\
\hline pBG1787 & $\begin{array}{l}\text { Derivative of pTRG in which } 150-477 \mathrm{bp} \text { nif } X \text { fragment released from BamHI-EcoRI } \\
\text { digested pBG1782 was cloned into the BamHI-EcoRI site of pTRG to generate an in-frame } \\
\alpha \text {-RNAP:C2NifX translation fusion }\end{array}$ & This study \\
\hline pMH5000 & $\begin{array}{l}\text { Derivative of PCR } 2.1 \text { TOPO in which } 477 \text { bp nif } X \text { fragment was cloned directly after PCR } \\
\text { using appropriate olignonucleotides designed with flanking BamHI-EcoRI sites }\end{array}$ & This study \\
\hline pMH5002 & $\begin{array}{l}\text { Derivative of pTRG in which } 477 \text { bp nifX fragment released from BamHI-EcoRI digested } \\
\text { pMH5000 was cloned into the BamHI-EcoRI site of pTRG to generate an in-frame } \alpha \text { - } \\
\text { RNAP:NifX translation fusion }\end{array}$ & This study \\
\hline pMH6000 & $\begin{array}{l}\text { Derivative of PCR } 2.1 \text { TOPO in which } 1509 \text { bp nifB fragment was cloned directly after PCR } \\
\text { using appropriate olignonucleotides designed with flanking EcoRI-BamHI sites }\end{array}$ & This study \\
\hline pMH6002 & $\begin{array}{l}\text { Derivative of pBT in which } 1509 \text { bp nifB fragment released from EcoRI-BamHI digested } \\
\text { pMH6000 was cloned into the EcoRI-BamHI site of pBT to generate an in-frame } \lambda \text { CI:NifB } \\
\text { translation fusion }\end{array}$ & This study \\
\hline pBG1785 & $\begin{array}{l}\text { Derivative of PCR } 2.1 \text { TOPO in which } 732 \mathrm{bp} \text { nafY fragment was cloned directly after PCR } \\
\text { using appropriate olignonucleotides designed with flanking EcoRI-BamHI sites }\end{array}$ & This study \\
\hline pBG1790 & $\begin{array}{l}\text { Derivative of } \mathrm{pBT} \text { in which } 732 \mathrm{bp} \text { nafY fragment released from EcoRI-BamHI digested } \\
\text { pBG1785 was cloned into the EcoRI-BamHI site of pBT to generate an in-frame } \lambda \text { CI:NafY } \\
\text { translation fusion }\end{array}$ & This study \\
\hline
\end{tabular}

enzyme digestions, agarose gel electrophoresis, ligations and $E$. coli transformation were carried out as described in the laboratory manual or according to the manufacturer's protocol ${ }^{[37,38]}$. Oligonucleotides used for PCR amplification were purchased from Integrated DNA Technologies (Coralville, IA). Following are the primers used to PCR amplify required gene fragments from $A$. vinelandii cells for cloning into pTRG or $\mathrm{pBT}$ vectors of the BacterioMatch Two Hybrid System ${ }^{\mathrm{TM}}$ :
(1) nifX (pTRG) (i) 5' GGA TCC ATG TCC AGC CCG ACC GGC 3'. (2) nifB (pBT) (i) 5' GAA TTC AGA ACT GAG CGT ACT TGG GCA AAA CAA T 3' and (ii) 5'GGA TCC TCA GGC CTT GGC CTG CAG CAG GGC T 3' (3) nifN (pBT) (i) 5' ATG GCC GAG ATC ATC AAT CGC AAC AAG GCC 3' and (ii) 5' GGA TCC TCA GTG CCT CCA TTG CGG CTG TTC GGT TGC CGG 3' (4) nafY (pBT) (i) 5'GAA TTC AGT AAC CCC CGT GAA 
CAT GAG TCG CGA 3' (ii) 5' GGA TCC TCA TGC CCT GGC CGC CTC GTC CTC GTC 3' (5) First 300 bp of nif $X$ encoding N-terminal NifX (pTRG) (i) 5, GGA TCC ATG TCC AGC CCG ACC CGA CAA TTG CAG GTA 3' (ii) 5' GAA TCC GGC CAT CAA CTG GCG CAC CGC CGA GGC GCC 3' (6) Last 324 bp of nifX encoding C-terminal NifX (pTRG) (i) 5'GGA TCC CGC TCC CAG CTC CTC TCG GTC GTC GAG TTC 3' (ii) GAA TTC CTA TTC GTC CCA GCC TTC GGC GGC CAT GGC 3'. In all the above oligonucleotides, the underlined sequences represent restriction enzyme recognition sites that were introduced during primer design so as to facilitate cloning of the DNA fragments into unique sites of the pBT or pTRG vectors. 'GGA TCC' is the BamHI recognition sequence and 'GAA TTC' is the EcoRI recognition sequence. The bacterial two-hybrid vectors pBT bait and pTRG target, containing the $\lambda \mathrm{CI}$ and $\alpha-$ RNAP domains, respectively, were the starting plasmids used for cloning the various gene fragments. Each gene fragment was PCR amplified using specific oligonucleotides as listed above and cloned into PCR 2.1 TOPO (Invitrogen, Carlsbad, CA) by ligation and transformation. These plasmids were then digested with EcoRI and BamHI and the resulting fragments were purified and ligated to the EcoRI-BamHI digested pBT or pTRG vectors. The ligated mixtures were used to transform E. coli XL1-Blue cells and desired clones were obtained and verified from the subsequent transformants by DNA isolation and restriction digestion analysis. The verified clones were designated as pMH5002 ( $\alpha$-RNAP + NifX), pBG1786 ( $\alpha$-RNAP + N-terminal (N1) NifX), pBG1787 ( $\alpha$-RNAP +Cterminal (C2) NifX), pMH6002 $(\lambda \mathrm{CI}+\mathrm{NifB})$, pBG1778 $(\lambda \mathrm{CI}+\mathrm{NifN})$ and pBG1790 $(\lambda \mathrm{CI}+\mathrm{NafY})$. The construction of the plasmids pBG1716 $(\alpha-$ RNAP + NifK), pBG1718 $(\lambda \mathrm{CI}+\mathrm{NifK})$ and pBG1866 $(\lambda \mathrm{CI}+$ $\mathrm{NifH})$ has been described elsewhere ${ }^{[39,40]}$. Subsequently, desired combinations of plasmids (e.g. pTRG-NifX + pBT-NafY) were used for cotransformation of E. coli XL1-Blue cells containing the reporter cassette. The resultant cotransformants were inoculated for performing $\beta$-galactosidase activity assays.

$\boldsymbol{\beta}$-Galactosidase assay: The $\beta$-galactosidase activity assay was performed as described under 'Molecular cloning ${ }^{\text {[38] }}$. Briefly, single E. coli transformants were inoculated into $5 \mathrm{~mL}$ of $2 \mathrm{YT}$ media supplemented with $34 \mu \mathrm{g} \mathrm{mL} L^{-1}$ of chloramphenicol and $5 \mu \mathrm{g} \mathrm{mL}{ }^{-1}$ of tetracycline. The cells were then incubated overnight at $37^{\circ} \mathrm{C}$ with shaking at $250 \mathrm{rpm}$. Then $200 \mu \mathrm{L}$ of the overnight culture was diluted into $5 \mathrm{~mL}$ of the same media and incubated at $37^{\circ} \mathrm{C}$ with shaking, until $\mathrm{OD}_{600}$ was between 0.3 and 0.6 (initial $\mathrm{OD}_{600}$ was 0.05 ). Cells from $1.5 \mathrm{~mL}$ of the culture were collected by centrifugation and resuspended in $500 \mu \mathrm{L} \mathrm{Z}$-buffer ${ }^{[38]}$. The resuspended cells were lysed by adding $50 \mu \mathrm{L}$ of chloroform and $25 \mu \mathrm{L}$ of $0.1 \%$ SDS and kept at $28^{\circ} \mathrm{C}$ with shaking for 5-10 min. To measure the $\beta$ galactosidase activity from the cell lysate, $900 \mu \mathrm{L}$ of Zbuffer- $\beta$-mercaptoethanol solution $(0.27 \mathrm{~mL}$ of $\beta$ mercaptoethanol per $100 \mathrm{~mL}$ of Z-buffer) was added to $100 \mu \mathrm{L}$ culture followed by $200 \mu \mathrm{L}$ Z-buffer/ONPG (onitrophenyl- $\beta$-D-galactopyranoside $-4 \mathrm{mg}$ ONPG per $\mathrm{mL}$ of Z-buffer). The time of ONPG addition was recorded and the tubes were incubated at $37^{\circ} \mathrm{C}$. When yellow color was visible, $500 \mu \mathrm{L}$ of $1 \mathrm{M} \mathrm{Na}_{2} \mathrm{CO}_{3}$ was added to each tube to terminate the reaction and the time was recorded. The optical densities at $420 \mathrm{~nm}$ as well as $550 \mathrm{~nm}$ were recorded. The $\beta$-galactosidase units were defined as the amount of enzyme which hydrolyzes $1 \mu \mathrm{mol}$ of ONPG to o-nitrophenol and Dgalactose per minute. $\beta$-Galactosidase activity in Miller Units was calculated as $1000 \times\left[\mathrm{OD}_{420}-\left(1.75 \times \mathrm{OD}_{550}\right) /\left(t \times V \times \mathrm{OD}_{600}\right)\right]$, where $t$ is the elapsed time (in min) of incubation, $V$ is the 0.1 $\mathrm{mL} \times$ concentration factor and $\mathrm{OD}_{600}$ is the absorbance of $1 \mathrm{~mL}$ of culture at $600 \mathrm{~nm}$.

Structural homology modeling: The Swiss PDB software (freely available online) ${ }^{[41]}$ was used for homology modeling and 3D representation of the derived NifX protein structure. The NafY structure (PDB ID: 1P90) was used as the template for superimposing the raw NifX amino acid sequence and deriving its $3 \mathrm{D}$ structure.

\section{RESULTS AND DISCUSSION}

Interaction of NifB with NifX. The BacterioMatch $^{\mathrm{TM}}$ Two-Hybrid System was used to determine which proteins in the FeMoco maturation pathway directly interacted with NifX. The NifB protein provides a Fe-S precursor, termed NifB-co, for FeMoco assembly ${ }^{[18,19]}$. Since the nifB gene product was found to be essential for the functioning of alternative nitrogenases (Mo-independent) also, therefore it is thought that the NifB-co represents the Fe-S primary cluster for both, the synthesis of FeMoco and the cofactors of alternative nitrogenases ${ }^{[18,42]}$. It is believed that the NifB-co is transferred from NifB to NifEN, which acts as a site for rearrangement of the primary $[\mathrm{Fe}-\mathrm{S}]$ to form the $[\mathrm{Fe}-\mathrm{S}]$ core of the FeMoco, probably after a structural contact between NifEN and 
$\mathrm{NifH}^{[19]}$. The NifB protein sequence comprises of two different domains: an N-terminal SAM radical domain and a C-terminal NifX-like domain, that is a domain present in certain FeMoco binding proteins ${ }^{[43]}$. The presence of the C-terminal NifX-like domain could indicate that this region in NifB is either involved in binding NifB-co precursors and forming the NifB-co or binding a preformed NifB-co for transfer to another protein in the FeMoco biosynthetic pathway ${ }^{[44]}$.

Although it was evident from previous reports that NifX is capable of binding to NifB-co in one of the stages during FeMoco biosynthesis ${ }^{[30]}$, a direct proteinprotein interaction between the NifB and NifX proteins had not been demonstrated before. Our results support the idea that there is an interaction between NifB and NifX as found by measuring the $\beta$-galactosidase activity of $E$. coli cotransformants harboring the plasmids pMH6002 ( $\lambda$ CI:NifB) and pMH5002 (RNAP:NifX). As compared to the $\beta$-galactosidase activity obtained from $E$. coli cotransformants harboring only the $\mathrm{pBT}$ and pTRG plasmids (negative control--22.93 \pm 6.12 Miller Units), the activity obtained from cells harboring pMH6002 and pMH5002 was much higher $(49.7 \pm 9.11$ Miller Units), indicating a protein-protein interaction between NifB and NifX (Table 2). Since the NifB and NafY proteins share many similarities between their amino acid sequences and since NafY was shown to bind to the apodinitrogenase $^{[35]}$, we also examined the ability of NifB and NifK to interact with each other and found that the $\beta$-galactosidase activity of $E$. coli cotransformants harboring the plasmids pMH6002 ( $\lambda$ CI:NifB) and pBG1716 (RNAP:NifK) was $20.47 \pm$ 4.78 Miller Units, suggesting lack of interaction between NifB and NifK.

Interaction of NafY with NifX: A new family of iron and molybdenum (or vanadium) cluster-binding proteins comprising of NifY, NifX, VnfX and NafY was described by Rubio et. al in $2002^{[25]}$. The $A$. vinelandii NafY protein (nitrogenase accessory factor $\mathrm{Y}$, also known as $\gamma$, was reported to be a molecular chaperone that assisted in maintaining the apodinitrogenase in a conformation that facilitated the insertion of the FeMoco by acting as a metalloinsertase $^{[25]}$. Mutational analysis of the strains containing mutations in both naf $Y$ and nif $X$ showed that these were severely affected in diazotrophic growth, indicating their physiological importance in the stabilization of the apodinitrogenase $\mathrm{e}^{[25]}$. Subsequently, the 3-dimensional structure of the NafY protein from $A$. vinelandii revealed two distinct domains of this protein: an N-terminal (Met1 to Leu98) domain and a Cterminal (Glu99 to Ser232) 'core' domain ${ }^{[35]}$. The NafY core domain was shown to be capable of binding the FeMoco of nitrogenase but unable to bind to apodinitrogenase in the absence of the N-terminal domain $^{[35]}$.

In a study that involved utilization of ${ }^{99} \mathrm{Mo}$ and a purified in vitro FeMo-co biosynthesis system for investigating the incorporation of the ${ }^{99}$ Mo radiolabel into proteins involved in the biosynthesis of FeMo-co, it was found that there was transfer of the ${ }^{99} \mathrm{Mo}$ label from NifH and NifX to NafY or apodinitrogenase ${ }^{[31]}$. In light of the earlier experiments, our data strengthens the role of NifX as a transporter of a fully formed FeMoco cluster to NafY. Our work helped in detecting a direct protein-protein interaction between NifX and NafY, as evident from the $\beta$-galactosidase activity $(42.49 \pm 8.62$ Miller Units) of $E$. coli cells harboring the plasmids pMH5002 (RNAP:NifX) and pBG1790 ( $\lambda$ CI:NafY) (Table 2). Through earlier studies, it was predicted that the NifX was involved in the latter part of the biosynthetic pathway of FeMoco formation ${ }^{[31]}$. Our results confirmed the above prediction, because we were able to identify an interaction between NifX with NafY but neither between NifX and NifN or NifX and $\mathrm{NifH}$, both of which are involved in the initial steps of the pathway. The $\beta$-galactosidase activity corresponding to the interaction between $\mathrm{NifH}$ and NifX was $32.98 \pm 6.67$ Miller Units and between NifN and NifX was $31.32 \pm 6.05$ Miller Units, indicating absence of any interaction between these pairs of proteins, when compared to the negative and positive controls $(22.93 \pm 6.12$ and $115.59 \pm 25.01$ Miller Units respectively) (Table 2). We also tested the validity of the results obtained through the BacterioMatch ${ }^{\mathrm{TM}}$ Two Hybrid system by determining whether the NafY and NifK proteins, that have been reported to associate with each other based on immunoblot analyses in earlier studies $^{[35]}$, showed evidence of interaction with each other by this method. Our results established that a strong interaction exists between the NafY and NifK proteins $(55.71 \pm 9.30$ Miller Units $)$ and that this bacterial two-hybrid method was indeed reliable for our experimental objectives (Table 2).

Interaction of NifDK (fusion) and NifK with NifX: A role for NifX in the insertion process of the FeMoco into apodinitrogenase has been suggested earlier ${ }^{[31]}$. In that study, the in vitro FeMoco biosynthesis assay was performed and the incorporation of ${ }^{99} \mathrm{Mo}$ radiolabel into apodinitrogenase was examined in reactions including and excluding NifX. It was found that the minus NifX 
Am. J. Biochem. \& Biotechnol., 3 (2): 96-106, 2007

\begin{tabular}{|c|c|c|c|}
\hline $\begin{array}{l}\text { Plasmid to which } \lambda \text { CI } \\
\text { translationally fused }\end{array}$ & $\begin{array}{l}\text { Plasmid to which } \alpha \text {-RNAP was } \\
\text { translationally fused }\end{array}$ & $\begin{array}{l}\beta \text {-Galactosidase } \\
(\text { Miller units) })^{\mathrm{a}}\end{array}$ & Interacting Peptides \\
\hline pBG1713 ( $\lambda$ CI: NifD-K fusion) & pMH5002 (RNAP: NifX) & $45.24 \pm 8.72$ & NifD-K and Nif $X$ \\
\hline pMH5007 ( $\lambda$ CI: NifD) & pMH5002 (RNAP: NifX) & $36.95 \pm 3.74$ & None \\
\hline pBG1718 ( $\lambda$ CI: NifK) & pMH5002 (RNAP: NifX) & $46.34 \pm 8.81$ & NifK and NifX \\
\hline pBG1718 ( $\lambda \mathrm{CI}:$ NifK) & pBG1786 (RNAP:N1NifX) & $33.93 \pm 8.74$ & None \\
\hline pBG1718 ( $\lambda$ CI: NifK) & pBG1787 (RNAP:C2NifX) & $35.50 \pm 7.81$ & None \\
\hline pBG1778 ( $\lambda \mathrm{CI}: \mathrm{NifN})$ & pMH5002 (RNAP: NifX) & $31.32 \pm 6.05$ & None \\
\hline PBG1866 ( $\lambda$ CI: NifH) & pMH5002 (RNAP: NifX) & $32.98 \pm 6.67$ & None \\
\hline pMH6002 ( $\lambda$ CI: NifB) & pMH5002 (RNAP: NifX) & $49.7 \pm 9.11$ & NifB and NifX \\
\hline pMH6002 ( $\lambda$ CI: NifB) & pBG1716 (RNAP: NifK) & $20.47 \pm 4.78$ & None \\
\hline pBG1790 ( $\lambda$ CI: NafY) & pMH5002 (RNAP: NifX) & $42.49 \pm 8.62$ & NafY and NifX \\
\hline pBG1790 ( $\lambda$ CI: NafY) & pBG1716 (RNAP: NifK) & $55.71 \pm 9.30$ & NafY and NifK \\
\hline pBT & pMH5002 (RNAP: NifX) & $29.87 \pm 1.63$ & None \\
\hline pBT & PTRG & $22.93 \pm 6.12$ & None \\
\hline pBT-LGF2 & pTRG-Gal11 ${ }^{\mathrm{p}}$ & $115.59 \pm 25.01$ & $\begin{array}{l}\text { Mutant form of Gall protein } \\
\text { with Gal11 }\end{array}$ \\
\hline
\end{tabular}

${ }^{a}$ Each assay was performed a minimum of four times for accuracy and the $\beta$-galactosidase activity units shown are an average of three independent observations.

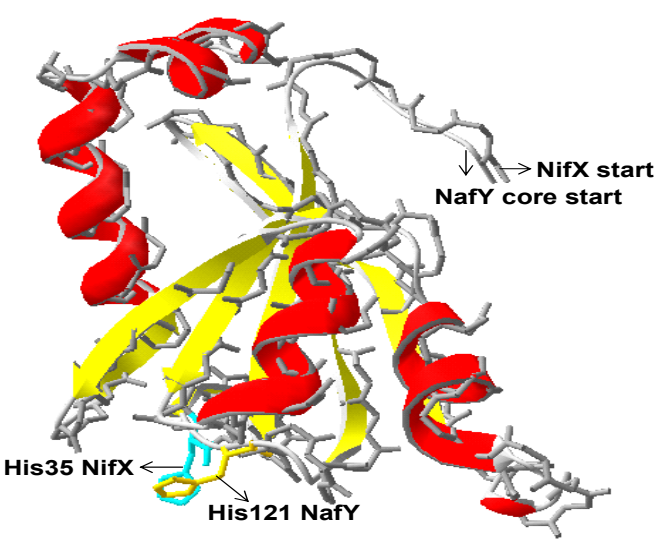

Fig. 1: Homology modeling of NifX using NafY core structure. The NafY core domain structure (PDB ID: 1P90) was used as a template for homology modeling of NifX. The NafY structure is represented in ribbons and the NifX structure in backbone. The NafY core domain start (Glu99) and NifX start (Met 1) are indicated by arrows. The His121 ligand of NafY that is known to be important for FeMoco binding (11) is shown to coincide with the His 35 residue of NifX

reaction showed lower levels of ${ }^{99} \mathrm{Mo}$ label incorporated into dinitrogenase in comparison to the NifX containing reaction ${ }^{[31]}$. Evidence obtained through our two-hybrid data clearly indicates the presence of protein-protein interaction between the NifDK protein encoding a single fused unit of dinitrogenase ${ }^{[45]}$ and NifX and between the NifK component of the dinitrogenase and NifX. To further analyze if the NifX protein possessed specific or separate domains that helped in its interaction with $300 \mathrm{bp}$ of nif $X$, encoding the N-terminal half of the NifX protein (N1X) and the region spanning 216-477 bp of nifX, encoding the C- terminal half of NifX fusion) and pMH5002 ( $\alpha$ RNAP:NifX) was $45.24 \pm 8.72$ Miller Units and that of pBG1718 ( $\lambda$ CI: NifK) and pMH5002 ( $\alpha$-RNAP:NifX) was $46.34 \pm 8.81$. The $\beta$-galactosidase activity of the positive control that included cotransformed plasmids encoding two known interacting proteins - a mutant form of Gall protein and the Gal1 $1^{\mathrm{P}}$, fused to $\lambda \mathrm{CI}$ of pBT and $\alpha$-RNAP of pTRG respectively, was $115.59 \pm$ 25.01 Miller Units and value for the negative control that included cotransformed plasmids $\mathrm{pBT}$ and pTRG only was $22.93+6.12$ Miller Units. The $\beta$ galactosidase activity corresponding to interaction between NifD and NifX was $36.95 \pm 3.74$ Miller Units, suggesting that there was much lesser or no interaction between the two proteins (Table 2). To further analyze if the NifX protein possessed specific or separate domains that helped in its interaction with $300 \mathrm{bp}$ of nif $X$, encoding the $\mathrm{N}$-terminal half of the NifX protein (N1X) and the region spanning 216-477 bp of nifX, encoding the C-terminal half of NifX the NifK protein, we cloned the region spanning 1-300 bp of nif $X$, encoding the N-terminal half of the NifX protein (N1X) and the region spanning $216-477 \mathrm{bp}$ of nif $X$, encoding the C-terminal half of NifX (C2X), into the pTRG plasmid. The $\beta$-galactosidase activity indicating the extent of interaction between NifK and NifN1X was $33.93 \pm 8.74$ Miller Units and between NifK and NifC2X was $35.50 \pm 7.81$ Miller Units (Table 2). Thus, the N-terminal and C-terminal NifX domains were unable to retain the ability to interact with the NifK protein individually, indicating that either the entire NifX protein is essential for interaction with NifK or a domain comprising an overlapping region from the N- 


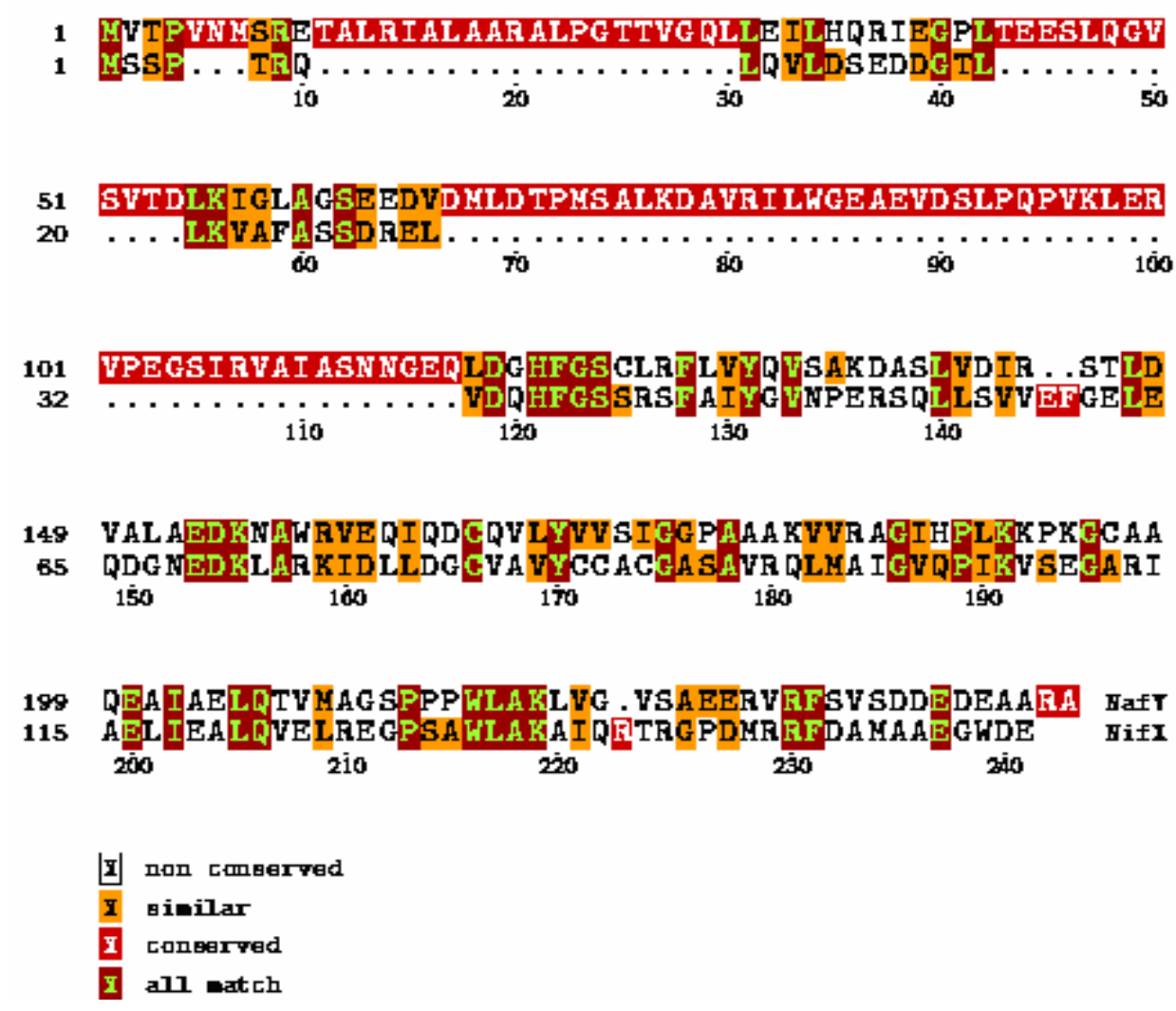

Fig. 2: ClustalW alignment of NifX and NafY protein sequences from $A$. vinelandii

terminal and C-terminal domains contains the necessary stretch of residues for interacting with NifK.

The NifX family of proteins comprises of proteins like NifB, NifY, NafY, VnfX and NifX and is based on the presence of a FeMoco-cluster binding domain. As seen in Fig. 1., the NifX protein is largely similar to the $\mathrm{C}$-terminal domain of the NafY protein. The C-terminal NafY protein known as the 'core' domain, was shown to be capable of binding to the FeMoco but unable to bind to the apodinitrogenase in the absence of the first domain, indicating that the $\mathrm{N}$-terminal region of $\mathrm{NafY}$ was important in interacting with dinitrogenase ${ }^{[35]}$. Our studies have demonstrated that the NifX is capable of interacting with the NifK protein. The fact that the NifX does not have a domain corresponding to the Nterminal region of $\mathrm{NafY}$ and yet is able to interact with NifK could be explained as a possible conservation of a few residues at the beginning of the NifX protein that are important in maintaining an interaction with NifK (Fig. 2). It could also be that whereas NafY interacts with a different region of the dinitrogenase protein, the NifX acts upon yet another separate region of the same protein and therefore the NifX protein which could actually be considered as only the C-terminal half of $\mathrm{NafY}$, may be involved in the dual role of FeMoco binding and dinitrogenase binding but awaits specific evidence regarding the residues involved.

Through this work, utilizing a simple two-hybrid approach, we have been able to reveal certain important interactions between the NifX protein and some other proteins related to the FeMoco biosynthetic pathway of nitrogenase. Mainly, the following interactions were found: (i) NifB and NifX (ii) NafY and NifX (iii) NifDK(fusion) and NifX (iv) NifK and NifX. Further protein-protein interaction based studies involving NifU, NifS, NifQ and NifV with NifX would certainly prove useful in better deciphering the role of NifX and the events of the FeMoco biosynthetic pathway as a whole. However, in light of our current findings and several previous insights into the FeMoco biosynthetic pathway, we propose a model that best explains the order of events that occurs during the FeMoco biosynthesis, assembly and insertion. It should be noted that there exist several different views of the exact 


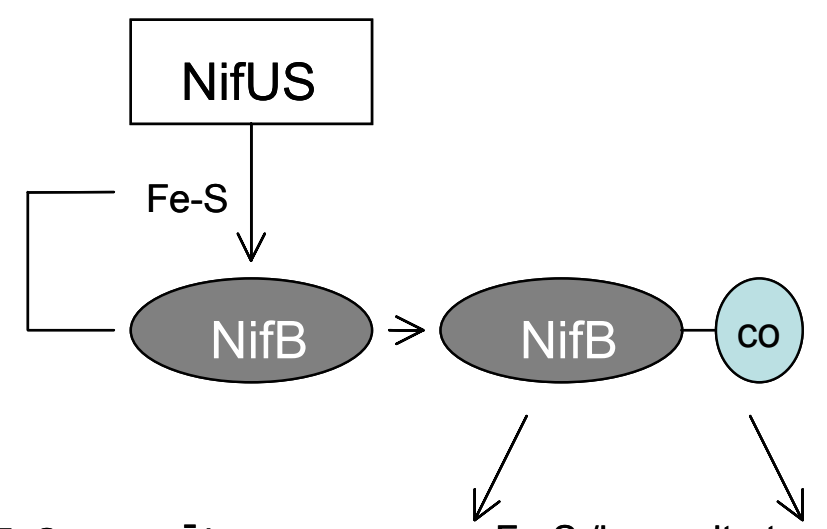

FeS process̄ing

Fe-S /homocitrate

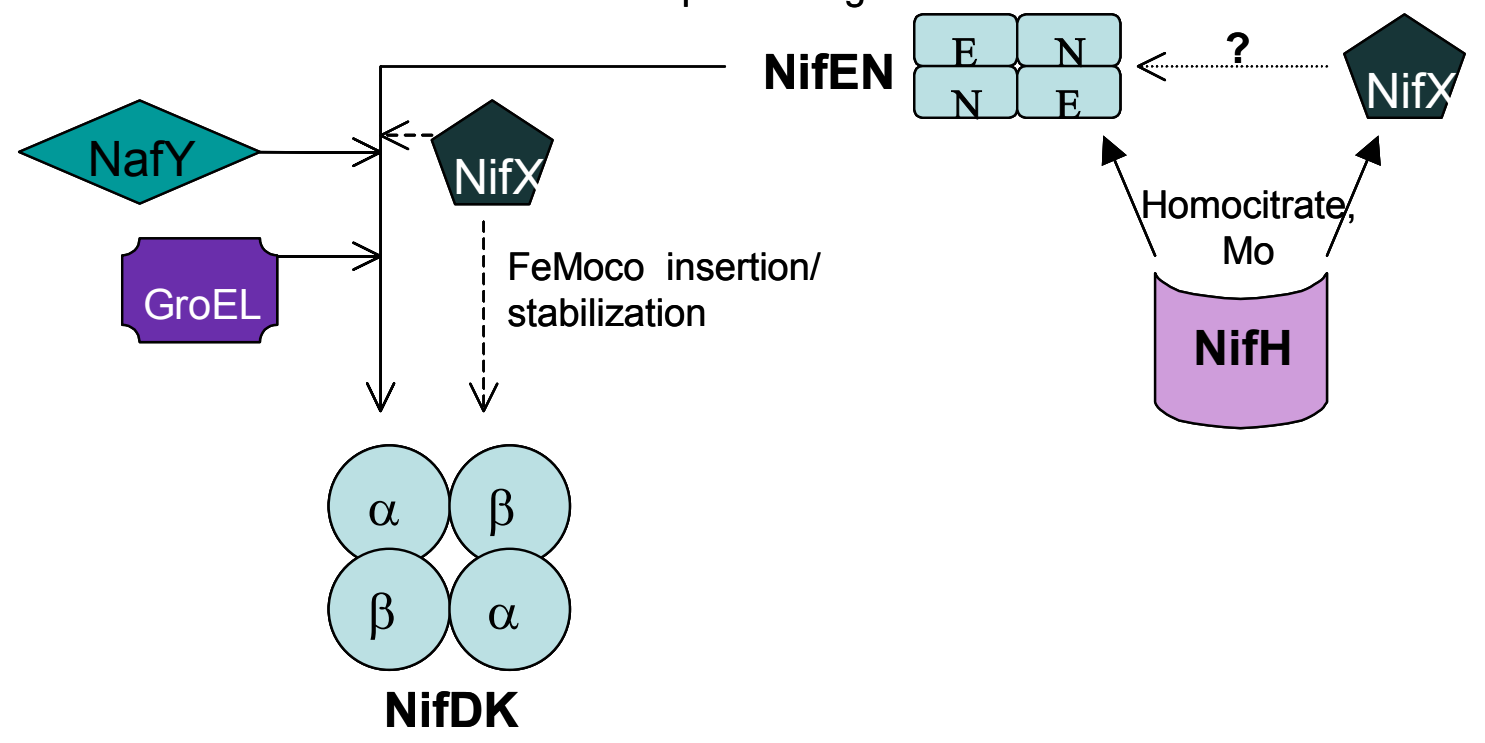

Fig. 3: Proposed model for the role of NifX in FeMoco biosynthesis/insertion pathway. The NifU and NifS proteins participate in the initial Fe/S mobilization; this precursor Fe-S moiety is then transferred to the NifB protein, which is then termed as NifB-co. The NifX protein may play a role as a molybdenum (Mo) carrier from the NifB to the NifEN complex or it may provide the homocitrate moiety to NifEN. This processed FeMoco is inserted and stabilized in the NifDK $(\mathrm{MoFe})$ protein and this activity is assisted by NifX, NafY and GroEL

sequence of events in the FeMoco biosynthetic pathway due to the complexity of the system and possible redundant functions of the involved proteins, causing difficulty in assigning confirmed roles. As seen in our suggested model in Fig. 3, the FeMoco biosynthetic pathway starts with the initial mobilization and assembly of the Fe-S fragments and is carried out by the NifUS complex ${ }^{[26]}$; This Fe-S fragment is then delivered to NifB and a Fe-S core is formed on it ${ }^{[18]}$. Following this, the core is transferred from NifB to the NifEN complex. Based on previous studies and the detection of an interaction between NifB and NifX in our study, it could be possible that NifX facilitates the transfer of the core from NifB to NifEN ${ }^{[30]}$. It is possible that the addition of homocitrate takes place at this step, since one previous study has shown that the NifX may be involved in specifying the organic acid moiety of the FeMoco precursor ${ }^{[31]}$. Following this, multiple FeMoco processing steps take place on the NifEN complex, which acts as a scaffold for this purpose $^{[7,8]}$. Very recently, Corbett et. al showed through x-ray absorption spectroscopy studies that the first isolatable FeMoco precursor on NifEN was a molybdenum-free analog of FeMoco and that the molybdenum was probably incorporated afterwards at the position previously occupied by the non-cysteine ligated terminal iron atom ${ }^{[46]}$. As found in other recent studies, the NifH may perform the role of facilitating the insertion of Mo and homocitrate into the FeMoco, probably after MgATP hydrolysis ${ }^{[47]}$. The GroEL 
protein is also required for the final assembly of the MoFe protein; It functions as a molecular chaperone for insertion of FeMoco into the MoFe protein ${ }^{[48]}$. It could be possible that the MgATP hydrolysis is actually necessary for the function of GroEL in the final assembly reaction and has no significant link with the NifH protein ${ }^{[48]}$. As found in our studies, the interaction of NifX with NafY indicates that the NifX may again function as an escort protein at this step and participate in the transfer of the intermediate or completed FeMoco cluster to the NafY protein. In the following steps, the assembled FeMoco that has been delivered to the NafY protein is carried to the apo-dinitrogenase. It has been suggested that the NafY protein dissociates from the MoFe protein after the final insertion process in completed. Since we have detected an interaction between the NifX and NifK proteins in this work, we propose that the NifX may perform a similar role to that of NafY and the final insertion of the FeMoco into the apo-dinitrogenase may involve the participation of NifX. It may also be possible that the NifX stabilizes the apo-dinitrogenase in a conformation suitable for FeMoco insertion through its interaction with NifK. There have been earlier speculations concerning the overlapping or redundant roles of the proteins involved in the FeMoco biosynthetic pathway, for example, the role of NifY was found to be similar to that of NafY in studies involving the FeMoco biosynthetic pathway in $K$. pneumoniae and $A$. vinelandii ${ }^{[49]}$. However, this occurrence has not been yet explainable and future studies that investigate the FeMoco biosynthetic pathway in detail may lead to meaningful answers.

\section{ACKNOWLEDGMENTS}

Part of this work was done at Bowling Green State University, Ohio. This research is partly supported by NIH and NSF to NG and LP. This material was also based on work supported by the National Science Foundation, while NG working at the Foundation. We thank members of Gavini/Pulakat laboratory for helpful discussions and technical help.

\section{REFERENCES}

1. Barney, B.M., H.I. Lee, P.C. Dos Santos, B.M., Hoffman, D.R. Dean and S.C. Seefeldt, 2006. Breaking the $\mathrm{N}_{2}$ triple bond: insights into the nitrogenase mechanism. Dalton Trans., 19: 227784.

2. Igarashi, R.Y. and L.C. Seefeldt, 2003. Nitrogen fixation: the mechanism of the Mo-dependent nitrogenase. Crit. Rev. Biochem. Mol. Biol., 38: 351-384.
3. Rees, D.C., F.A. Tezcan, C.A. Haynes, M.Y. Walton, S. Andrade, O. Einsle and J.B. Howard, 2005. Structural basis of biological nitrogen fixation. Philos. Trans. R. Soc. London A, 363: 971-984.

4. Howard, J.B. and D.C. Rees. 1996. Structural basis of biological nitrogen fixation. Chem. Rev., 96: 2965-2982.

5. Chan, M.K., J. Kim and D.C. Rees, 1993. The nitrogenase FeMo-cofactor and P-cluster pair: 2.2 A resolution structures. Science, 260: 792-794.

6. Einsle, O., F.A. Tezcan, S.L. Andrade, B. Schmid, M. Yoshida, J.B. Howard and D.C. Rees, 2002. Nitrogenase MoFe-protein at 1.16 A resolution: A central ligand in the FeMo-cofactor. Science. 297: 1696-1700.

7. Dos Santos, P.C., D.R. Dean, Y. Hu and M.W. Ribbe, 2004. Formation and insertion of the nitrogenase iron-molybdenum cofactor. Chem. Rev., 104: 1159-1174.

8. Rubio, L.M. and P.W. Ludden, 2005. Maturation of Nitrogenase: a Biochemical Puzzle. J. Bacteriol., 187: 405-414.

9. Peters, J.W., K. Fisher and D.R. Dean, 1995. Nitrogenase structure and function: a biochemicalgenetic perspective. Annu. Rev. Microbiol., 49: 335-366.

10. Shah, V.K., P. Rangaraj, R. Chatterjee, R.M. Allen, J.T. Roll, G.P. Roberts and P.W. Ludden, 1999. Requirement of NifX and other Nif proteins for in vitro biosynthesis of the iron-molybdenum cofactor of nitrogenase. J. Bacteriol., 181: 2797-2801.

11. Rubio, L.M., S.W. Singer and P.W. Ludden. 2004. Purification and characterization of NafY (apodinitrogenase \{gamma\} subunit) from Azotobacter vinelandii. J. Biol. Chem., 279: 1973919746.

12. Imperial, J., V.K. Shah, R.A. Ugalde, P.W. Ludden and W.J. Brill, 1987. Iron-molybdenum cofactor synthesis in Azotobacter vinelandii nif mutants. J. Bacteriol., 169: 1784-1786

13. Ugalde, R. A., J. Imperial, V.K. Shah and W.J. Brill, 1984. Biosynthesis of iron-molybdenum cofactor in the absence of nitrogenase. J. Bacteriol., 159: 888-893.

14. Shah, V.K., J. Imperial, R.A. Ugalde, Ludden, P.W. and W.J. Brill, 1986. In vitro synthesis of the iron-molybdenum cofactor of nitrogenase. Proc. Natl. Acad. Sci. USA, 83: 1636-1640.

15. Meijer, W.G. and F.R. Tabita, 1992. Isolation and characterization of the nifUSVW-rpoN gene cluster from Rhodobacter sphaeroides. J. Bacteriol., 174: 3855-3866. 
16. Paustian, T.D., V.K. Shah and G.P. Roberts, 1989. Purification and characterization of the nifN and nifE gene products from Azotobacter vinelandii mutant UW45. Proc. Natl. Acad. Sci. USA, 86: 6082-6086.

17. Robinson, A.C., D.R. Dean and B.K. Burgess, 1987. Ironmolybdenum cofactor biosynthesis in Azotobacter vinelandii requires the iron protein of nitrogenase. J. Biol. Chem., 262:14327-14332

18. Shah, V.K., J.R. Allen, N.J. Spangler and P.W. Ludden, 1994. In vitro synthesis of the ironmolybdenum cofactor of nitrogenase. Purification and characterization of NifB cofactor, the product of NIFB protein. J. Biol. Chem., 269: 1154-1158.

19. Allen, R.M., R. Chatterjee, P.W. Ludden and V.K. Shah, 1995. Incorporation of iron and sulfur from NifB cofactor into the iron-molybdenum cofactor of dinitrogenase. J. Biol. Chem., 270: 2689026896.

20. Goodwin, P.J., J.N. Agar, J.T. Roll, G.P. Roberts, M.K. Johnson and D.R. Dean. The Azotobacter vinelandii NifEN complex contains two identical [4Fe-4S] clusters. Biochemistry, 37: 10420-10428.

21. Roll, J.T., V.K. Shah, D.R. Dean and G.P. Roberts, 1995. Characteristics of NIFNE in Azotobacter vinelandii strains. Implications for the synthesis of the iron-molybdenum cofactor of dinitrogenase. J. Biol. Chem., 270: 4432-4437.

22. Allen, R.M., R. Chatterjee, P.W. Ludden and V.K. Shah, 1996. The requirement of reductant for in vitro biosynthesis of the iron-molybdenum cofactor of nitrogenase. J. Biol. Chem., 271: 4256-4260.

23. Chatterjee, R., R.M. Allen, V.K., Shah and P.W. Ludden, 1994. Nucleotide and divalent cation specificity of in vitro iron-molybdenum cofactor synthesis. J. Bacteriol., 176: 2747-2750.

24. Homer, M.J., D.R. Dean and G.P. Roberts, 1995. Characterization of the $\gamma$ protein and its involvement in the metallocluster assembly and maturation of dinitrogenase from Azotobacter vinelandii. J. Biol. Chem., 270: 24745-24752.

25. Rubio, L.M., P. Rangaraj, M.J. Homer, G.P. Roberts and P.W. Ludden, 2002. Cloning and mutational analysis of the $\gamma$ gene from Azotobacter vinelandii defines a new family of proteins capable of metallocluster binding and protein stabilization. J. Biol. Chem., 277: 14299-14305.

26. Yuvaniyama, P., J.N. Agar, V.L. Cash, M.K. Johnson and D.R. Dean, 2000. NifS-directed assembly of a transient [2Fe-2S] cluster within the NifU protein. Proc. Natl. Acad. Sci. U.S.A., 97: 599-604
27. Zheng, L.M., R.H. White and D.R. Dean, 1997. Purification of the Azotobacter vinelandii nifVencoded homocitrate synthase. J. Bacteriol., 179: 5963-5966.

28. Imperial, J., R.A. Ugalde, V.K. Shah and W.J. Brill, 1984. Role of the nifQ gene product in the incorporation of molybdenum into nitrogenase in Klebsiella pneumoniae. J. Bacteriol., 158: 187-94

29. Kennedy, C. and D.R. Dean, 1992. The nifU, nifS and nif $V$ gene products are required for activity of all three nitrogenases of Azotobacter vinelandii. Mol. Gen. Genet., 231: 494-8.

30. Rangaraj, P., C. Ruttimann-Johnson, V.K. Shah and P.W. Ludden, 2001. Accumulation of ${ }^{55} \mathrm{Fe}-$ labeled precursors of the iron-molybdenum cofactor of nitrogenase on NifH and NifX of Azotobacter vinelandii. J. Biol. Chem., 276: 1596815974

31. Rangaraj, P. and P.W. Ludden, 2002. Accumulation of ${ }^{99}$ Mo-containing IronMolybdenum Cofactor Precursors of Nitrogenase on NifNE, NifH and NifX of Azotobacter vinelandii. J. Biol. Chem., 277: 40106-40111.

32. http://ca.expasy.org/uniprot/P14887

33. Anantharaman, V., E.V. Koonin and L. Aravind, 2001. Regulatory potential, phyletic distribution and evolution of ancient, intracellular smallmolecule-binding domains. J. Mol. Biol., 307: 1271-1292.

34. Jacobson, M.R., K.E. Brigle, L.T. Bennett, R.A. Setterquist, M.S. Wilson, V.L. Cash, J. Beynon, W.E. Newton and D.R. Dean, 1989. Physical and genetic map of the major nif gene cluster from Azotobacter vinelandii. J. Bacteriol., 171: 1017-27.

35. Dyer, D.H., L.M. Rubio, J.B. Thoden, H.M. Holden, P.W. Ludden and I. Rayment, 2003. The three-dimensional structure of the core domain of NafY from Azotobacter vinelandii determined at 1.8-A resolution. 278: 32150-6.

36. Dove, S.L., J.K. Joung and J.K.A. Hochschild, 1997. Activation of prokaryotic transcription through arbitrary protein-protein contacts. Nature, 386: 627-630.

37. BacterioMatch Two-Hybrid System Instruction Manual. 2001. Stratagene, Inc., La Jolla, CA.

38. Sambrook, J.F., E.F. Fritsch and T. Maniatis, 1992. Molecular Cloning: A Laboratory Manual, Cold Spring Harbor Laboratory, Cold Spring Harbor, New York. 
39. Verma, S., 2002. Investigation of the NifM-NifH interaction using BacterioMatch Two Hybrid System. Masters thesis. Bowling Green State University, Biological Sciences Department.

40. Lahiri, S., L. Pulakat and N. Gavini, 2005. Functional NifD-K fusion protein in Azotobacter vinelandii is a homodimeric complex equivalent to the native heterotetrameric MoFe protein. Biochem. Biophys. Res. Commun., 337: 677-684

41. Schwede, T., J. Kopp, N. Guex and M. Peitsch, 2003. SWISS-MODEL: An automated protein homology-modeling server. Nucl. Acids Res., 31: 3381-3385.

42. Joerger, R.D. and P.E. Bishop, 1988. Nucleotide sequence and genetic analysis of the nifB-nifQ region from Azotobacter vinelandii. J. Bacteriol., 170: 1475-1487.

43. Marchler-Bauer, A. and S.H. Bryant, 2004. CDSearch: protein domain annotations on the fly. Nucleic Acids Res., 32: 327-331.

44. Curatti, L., P.W. Ludden and L.M. Rubio, 2006. NifB-dependent in vitro synthesis of the ironmolybdenum cofactor of nitrogenase. Proc. Natl. Acad. Sci. USA, 103: 5297-5301.
45. Suh, M-H., L. Pulakat and N. Gavini, 2003. Functional expression of a fusion-dimeric $\mathrm{MoFe}$ protein of nitrogenase in Azotobacter vinelandii. J. Biol. Chem., 278: 5353-60.

46. Corbett, M.C., Y. Hu, A.W. Fay, M.W. Ribbe, B. Hedman and K.O. Hodgson, 2006. Structural insights into a protein-bound iron-molybdenum cofactor precursor. Proc. Natl. Acad. Sci. USA, 103: 1238-1243.

47. Hu, Y., A.W. Fay and M.W. Ribbe, 2005. Identification of a nitrogenase FeMo cofactor precursor on NifEN complex. Proc. Natl. Acad. Sci. USA, 102: 3236-3241.

48. Ribbe, M.W. and B.K. Burgess, 2001. The chaperone GroEL is required for the final assembly of the molybdenum-iron protein of nitrogenase. Proc. Natl. Acad. Sci. USA, 98: 5521-5525.

49. Homer, M.J., T.D. Paustian, V.K. Shah and G.P. Roberts, 1993. The nifY product of Klebsiella pneumoniae is associated with apodinitrogenase and dissociates upon activation with the ironmolybdenum cofactor. J. Bacteriol., 175: 49074910 . 